\title{
Downregulation of metastasis suppressor 1 (MTSS1) is associated with nodal metastasis and poor outcome in Chinese patients with gastric cancer
}

Ke Liu ${ }^{1 \dagger}$, Gefang Wang ${ }^{2+}$, Houzhong Ding ${ }^{3}$, Ying Chen ${ }^{4}$, Guanzhen Yu ${ }^{1 *}$, Jiejun Wang ${ }^{1 *}$

\begin{abstract}
Background: The putative tumor metastasis suppressor 1(MTSS1) is an actin-binding scaffold protein that has been implicated to play an important role in carcinogenesis and cancer metastasis, yet its role in the development of gastric cancer has not been well illustrated. In this study, we detected MTSS1 expression and explored its clinical significance in gastric cancer.
\end{abstract}

Methods: Immunohistochemistry was performed using tissue microarrays containing gastric adenocarcinoma specimens from 1,072 Chinese patients with normal adjacent mucosa, primary gastric cancer and lymph node (LN) metastasis and specific antibody against MTSS1. MTSS1 mRNA and protein expression were detected by reverse transcription-polymerase chain reaction and Western blotting. The clinical follow-up was done in the 669 patients living in Shanghai that was chose from the 1072 cases.

Results: Complete loss of MTSS1 expression was observed in 751 cases (70.1\%) of the 1,072 primary tumors and 103 (88\%) of 117 nodal metastases; and loss of MTSS1 expression was significantly associated with poorly differentiated tumors, large tumor size, deep invasion level, the presence of nodal metastases and advanced disease stage. Moreover, multivariate analysis demonstrated that loss of MTSS1 expression correlated significantly with poor survival rates $(\mathrm{RR}=0.194,95 \% \mathrm{Cl}=0.144-0.261, \mathrm{P}<0.001)$.

Conclusions: MTSS1 expression decreased significantly as gastric cancer progressed and metastasized, suggesting MTSS1 may serve as a useful biomarker for the prediction of outcome of gastric cancer.

\section{Background}

Gastric carcinoma (GC), which is the second most common cause of cancer-related death in the world, deprives more than 700,000 lives per annum [1]. Its incidence varies considerably worldwide and has recently been decreasing in developed countries, but remains stably in developing countries [2-4]. Furthermore, the fact that gastric cancer is insensitive to conventional chemotherapy and is rarely amenable to radiotherapy leaves the survival durations in patients with gastric cancer unchanged in recent years. This highlights the need for

\footnotetext{
*Correspondence: qiaoshanqian@yahoo.com.cn; jiejunw@csco.org.cn † Contributed equally

'Department of Medical Oncology, Changzheng Hospital, Shanghai, China Full list of author information is available at the end of the article
}

the determination of prognostic factors predicting the outcome and the development of novel therapeutic strategies. Previous studies have indicated that disease stage and lymph node metastasis are the most important prognostic factors in gastric cancer. Moreover, some molecular markers have been identified and attempted to use clinically [5-7]. Nevertheless, other potential prognostic factors related to survival in these patients remain unclear.

Metastasis suppressor 1 (MTSS1), also known as MIM (missing in metastasis), was originally identified by Lee et al [8] as a potential metastasis suppressor gene that was present in non-metastatic bladder cancer cell lines, but was not expressed in a metastatic bladder cancer cell line. This gene, mapped to human chromosome 
8q24.1, encodes a $5.3 \mathrm{~kb}$ mRNA and a polypeptide predicted to be an actin-binding protein of 356 amino acids with homology to the WASp (Wiscott-Aldrich Syndrome protein) family [8]. Functional analyses of MTSS1 have shown that MTSS1 induced actin-rich protrusions resembling microspikes and lamellipodia at the plasma membrane and promoted disassembly of actin stress fibres [9]. Actin filament assembly is associated with cytoskeletal structure organization and many forms of cell motility [10]. These data have suggested that MTSS1 protein may be important in regulating cytoskeletal dynamics, and as a consequence it would play a potential role in the invasion and metastatic behaviour of cancer cells.

The study surrounding MTSS1 is quite small, yet this protein has been the subject of controversy. Preliminary analysis by Northern blotting demonstrated that MTSS1 is widely expressed but is most abundant in spleen, thymus, testis, and prostate, with low levels also detected in uterus and colon [8]. Since this pioneering article, other reports have indicated that MTSS1 played a role as a metastasis suppressor in prostate cancer $[11,12]$, bladder cancer $[8,11,13]$ and benign lesions, but up-regulated in basal cell carcinomas [14]. However, other evidences showed that MTSS1 is unlikely to be a metastasis suppressor. It acts as a scaffold protein that interacts with actin-associated proteins to modulate lamellipodia formation [15]. Ma et al suggests that MTSS1 is a regulator of carcinogenesis in hepatocellular carcinoma [16]. And it is a member of the sonic hedgehog ( $\mathrm{SHH})$ signalling pathway that modulates Gli responses during growth and carcinogenesis [14].

Although these studies cited above suggested MTSS1 as a promising candidate biomarker and playing an important role in tumorigenesis, little is known about the function of MTSS1 in gastric cancer. In our study, we sought to determine the expression of MTSS1 in resected gastric cancers and investigate the correlation of MTSS1 expression and clinicopathologic features and survival, in an attempt to discover the potential influence of MTSS1 on the development of gastric cancer.

\section{Methods}

\section{Patient specimens}

A total of 1,072 patients with gastric cancer who underwent curative surgery at Changhai Hospital in Shanghai, People's Republic of China, from 2001 to 2005 were enrolled in this study. Mean age of these patients was 59 years old; 757(71\%) were male and 315 (29\%) were female. All the other clinicopathological characteristics of these patients could be found in previous study [17]. Clinical follow-up results were available for the 669 patients from the Shanghai area (mean follow-up duration, 40 months [range, 1-110 months]). Total number of death event was 380 cases, and 289 cases are still alive. All of the tissue specimens were obtained for the present study with patient informed consent, and the use of the human specimens was approved by the Changhai Hospital Institutional Review Board.

\section{Immunohistochemistry and evaluation of immunostaining} Tissue sections were deparaffinized in xylene, and then rehydrated in graded concentrations of ethyl alcohol (100\%, 95\%, 75\%, then water). TMA sections were microwave-treated twice in citrate buffer $\left(\mathrm{PH} \mathrm{6.0)}\right.$ at $99^{\circ}$ $\mathrm{C}$ for $6 \mathrm{~min}$. The sections were placed in $3 \% \mathrm{H}_{2} \mathrm{O}_{2}$ for $10 \mathrm{~min}$ to inhibit the endogenous peroxide activity, washed three times with phosphate-buffered saline (PBS) buffer for $5 \mathrm{~min}$ and placed in normal goat serum as blocking antibody at room temperature for $10 \mathrm{~min}$. The primary antibodies used were ab56780 (Abnova, Caltag-Medsystems Ltd., Buckingham, UK, 1:50) for MTSS1. After incubation at $4^{\circ} \mathrm{C}$ for $24 \mathrm{~h}$, sections were washed three times with PBS buffer for $10 \mathrm{~min}$. Biotinylated anti-mouse/rabbit immunoglobulin was used as the second antibody. 3, 3-Diaminobenzidine (DAB) was used as a chromogen. The sections were counter-stained with hematoxylin.

All slices were evaluated without knowledge of the clinical outcome. MTSS1 protein expression in the 1,072 cases was evaluated by two individuals (G. Y. and Y. C.) under an Olympus CX31 microscope (Olympus, Center Valley, PA). Sections were considered positive for MTSS1 when more than $5 \%$ of tumor cells were stained in the cell cytoplasm. Staining was scored independently by the two individuals who were blinded to each other's findings.

\section{RNA preparation and reverse transcription- polymerase chain reaction}

Total cellular RNA was isolated from the homogenised gastric samples using the AB gene Total RNA Isolation Reagent (Advanced Biotechnologies Ltd., Epsom, Surrey, UK). RNA concentration and quality were determined through spectrophotometric measurement (WPA UV 1101, Biotech Photometer, Cambridge, UK). cDNA was generated from 1 ug of each RNA sample and a reverse transcribed using a transcription kit (Takara, Kyoto, Japan). The quality of DNA was verified using $\beta$-actin primers (sense:GCTGTCACCTTCACCGTTC; antisense: CCATCGTCCACCGCAAAT). MTSS1 mRNA levels were assessed using MTSS1 primers: (sense: TGG GTCCACTGAGCCCCACACATTGTTG and antisense: GGTGgCCATtgTGgG GTGGAATG -AA). PCR amplification was carried out with Ex Taq DNA polymerase reaction system (Takara, Kyoto, Japan). Conditions for PCR were $94^{\circ} \mathrm{C} 4 \mathrm{~min}, 30 \mathrm{~s}$ at $94^{\circ} \mathrm{C}$ for denaturation, $30 \mathrm{~s}$ at $60^{\circ} \mathrm{C}$ for annealing and $90 \mathrm{~s}$ at $72^{\circ}$ 
$\mathrm{C}$ for elongation (30 cycles). PCR products were electrophoresed through $1.5 \%$ agarose gels and analyzed by computerized densitometric scanning of the images using the Quantity-One imaging software normalized with internal $\beta$-actin control.

\section{Western Blotting Assay}

Whole-cell lysates were prepared from human gastric cancer and normal gastric tissue specimens. Standard Western blotting was performed using a mouse monoclonal antibody against human MTSS1 in a 1:50 dilution (Abnova, Caltag-Medsystems Ltd., Buckingham, UK) and an anti-mouse/rabbit immunoglobulin (ZB-2305, Jackson, America). Equal protein sample loading was monitored by probing the same membrane filter with an anti- $\beta$-actin antibody. The probe proteins were detected using the Amersham enhanced chemiluminescence system according to the manufacturer's instructions.

\section{Statistical analysis}

Association among factors was evaluated by the Pearson $\chi 2$ test. Within-group correlations of continuous and ordinal variables were assessed using Pearson's correlation coefficient or Spearman's rank correlation coefficient when appropriate. Differences between samples mRNA and Western Blotting were assessed by paired t-test. Survival curves were calculated according to the Kaplan-Meier method. Survival data shown in this study were pertaining to overall survival. To this end, nongastric cancer deaths were considered as lost to follow up as of time of death in the statistical analysis. Differences between survival curves were examined with the log-rank test. Multivariate analysis of prognostic factors related to overall survival was carried out using Cox's proportional hazards model and a stepwise procedure. The covariates included sex, age, histological classification, Lauren's classification, tumor size, location of tumor, depth of invasion, lymph node metastasis, and disease stage. The accepted level of significance was $\mathrm{P}<$ 0.05 . Statistical analyses and graphics were performed with the SPSS 13.0 statistical package (SPSS, Inc., Chicago, IL).

\section{Results}

\section{MTSS1 expression in patients with gastric cancer}

All tissue microarray block sections selected for this study contained both normal and malignant epithelium. Figure 1 shows examples of tissue immunostained for the protein evaluated. MTSS1 immunostaining was of cytoplasm localization. The epithelium in normal mucosa specimens showed visible MTSS1 staining (Figure $1 \mathrm{~A}) .751$ cases $(70.1 \%)$ showed negative staining of MTSS1 and the other 321 cases showed positive staining. These results were in concordance with that in the
Western blotting study (Figure 1G). Importantly, we detected high expression of MTSS1 in adjacent normal epithelium but drastically reduced MTSS1 expression in the tumor cells (Figure 1F). Moreover, we observed that expression of MTSS1 varied with the different histological types. From the well and moderate histological levels to the histological undifferentiated, the expression of MTSS1 was gradually decreased (Figure 1B-D). In addition, MTSS1 expression was down-regulated in the liver metastases compared with the adjacent normal liver tissues, which serves as a positive control (Figure 1E).

Reverse transcription RT-PCR was used to evaluate MTSS1 expression level in 30 normal adjacent mucosa and 30 paired primary gastric cancer tissues. The result verified that MTSS1 expression level in GC tissues was significantly different from the paired normal adjacent mucosa. Consistent with the microarray data, this analysis showed that the MTSS1 expression mRNA level in $\mathrm{GC}$ was significantly lower than in normal adjacent mucosa. $(\mathrm{P}<0.001)$ (Figure $1 \mathrm{H})$.

\section{Correlation between MTSS1 expression and clinicopathologic characteristics of gastric cancer}

The GC patients were divided into two groups according to the MTSS1 expression levels:

MTSS1 low or negative expressers $(n=751)$ and MTSS1 positive expressers $(n=321)$. Correlation between MTSS1 expression level and clinicopathologic characteristics of GC is summarized in Table 1. A statistically significant association was observed between MTSS1 expression level and tumor size, histology and Lauren's classification. In 634 cases well-differentiated to moderately differentiated tumors, $235(37.1 \%)$ of the cases had positive MTSS1 expression in GC tissue; whereas in 438 poorly differentiated tumors, only 86 (19.6\%) presented positive MTSS1 expression ( $\mathrm{P}<$ 0.001 ). And in 618 cases of intestinal type in Lauren's classification, $211(34.1 \%)$ presented positive MTSS1 expression but 110 (24.2\%) cases with the diffuse-type showed positive. In the 825 cases of GC with tumor size $(<6 \mathrm{~cm}), 265(32.1 \%)$ presented MTSS1 expression, while only $56(22.7 \%)$ of 247 cases of GC with tumor size $(\geq 6 \mathrm{~cm})$ presented MTSS1 expression $(\mathrm{P}=$ 0.005).

We also observed that loss of MTSS1 expression correlated with advanced $\mathrm{T}, \mathrm{N}$, and TNM stage. Loss of MTSS1 expression occurred more frequently in large gastric tumors (invasion to level T3-T4) (76.1\%) than in small ones (level T0-T2) (55.4\%; P < 0.001) and more frequently in gastric tumors with regional $\mathrm{LN}$ metastasis $(76.3 \%)$ than in N0-stage tumors $(57.6 \%$; $\mathrm{P}<0.001)$. With regard to TNM stage, loss of MTSS1 expression was significantly associated with advanced disease stage: $78.8 \%$ at stage III -IV and 57\% at stage I- II $(\mathrm{P}<0.001)$. 

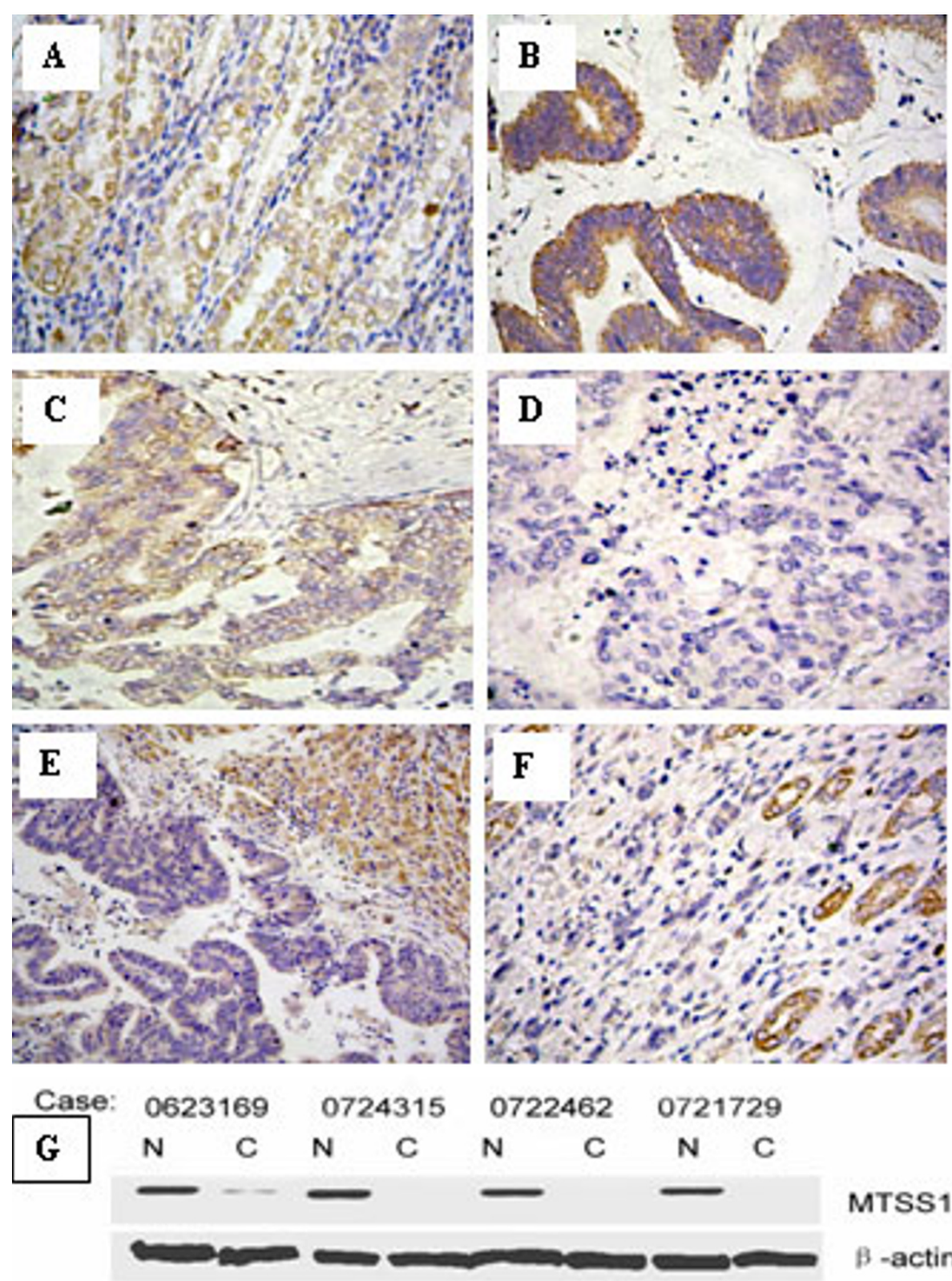

MTSS1

B -actin

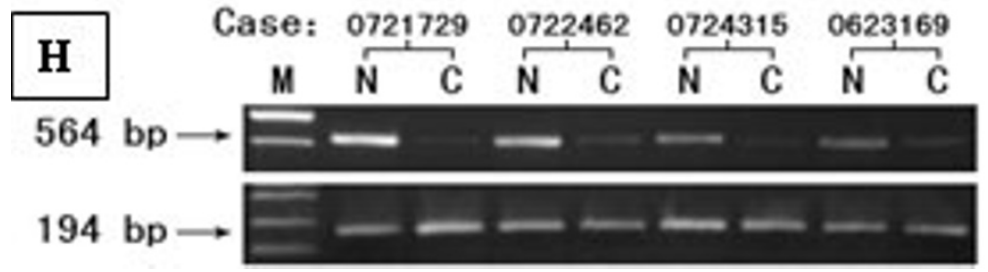

MTSS1

B-actin

Figure 1 Analysis of MTSS1 expression in human gastric cancers and adjacent normal mucosa specimens. A: Normal (nonneoplastic) gastric mucosa; B and C: Gastric cancer positive expression of MTSS1 in well-(B) and moderate-(C) differentiated tumors. D: Gastric cancer negative MTSS1 expression; E: MTSS1 expression in the hepatic metastasis and adjacent nonneoplastic liver tissues; F: MTSS1 expression in gastric cancer and adjacent nonneoplastic mucosa tissues; $\mathrm{G}$ and H: Western blotting analysis (G) and RT-PCR analysis (H) of MTSS1 in GC tissues and surrounding nontumor tissues, $\beta$-actin as a parallel control, N: surrounding nontumor tissues; T: tumor tissues. 
Table 1 Summary of clinicopathological parameters of gastric cancer and their correlation with loss of MTSS1 protein expression

\begin{tabular}{|c|c|c|c|}
\hline Features & No. of patients (\%) & Loss of MTSS1 expression (\%) & $P$ value \\
\hline Tumor size & & & 0.005 \\
\hline$<6 \mathrm{~cm}$ & $825(77.0 \%)$ & $560(67.9 \%)$ & \\
\hline$\geq 6 \mathrm{~cm}$ & $247(23.0 \%)$ & 191(77.3\%) & \\
\hline Site & & & NS \\
\hline Cardia and fundus & $180(16.8 \%)$ & $127(70.6 \%)$ & \\
\hline Corpus & $329(30.7 \%)$ & $229(69.6 \%)$ & \\
\hline Antrum & $519(48.4 \%)$ & $359(69.2 \%)$ & \\
\hline Whole & $44(4.1 \%)$ & $36(81.8 \%)$ & \\
\hline pT stage & & & $<0.001$ \\
\hline TO-T2 & $312(29.1 \%)$ & $173(55.4 \%)$ & \\
\hline $\mathrm{T} 3 / \mathrm{T} 4$ & $760(70.9 \%)$ & $578(76.1 \%)$ & \\
\hline pN stage & & & $<0.001$ \\
\hline NO & $356(33.2 \%)$ & $205(57.6 \%)$ & \\
\hline N1-3 & $716(66.8 \%)$ & $546(76.3 \%)$ & \\
\hline Disease stage & & & $<0.001$ \\
\hline$|/| \mid$ & 427(39.8\%) & $243(57 \%)$ & \\
\hline III/IV & $645(60.2 \%)$ & $508(78.8 \%)$ & \\
\hline Differentiation & & & $<0.001$ \\
\hline Well/Moderate & $634(59.1 \%)$ & $399(62.9 \%)$ & \\
\hline Poorly & $438(40.9 \%)$ & $352(80.4 \%)$ & \\
\hline Lauren's classification & & & $<0.001$ \\
\hline Intestinal-type & $618(57.6 \%)$ & $407(65.9 \%)$ & \\
\hline Diffuse-type & $454(42.4 \%)$ & $344(75.8 \%)$ & \\
\hline Total & 1072 & $751(70.1 \%)$ & \\
\hline
\end{tabular}

Relationship of MTSS1 protein expression in primary gastric tumors and LN metastases

In the tissue microarrays, 117 cases had available matched LN metastases. The MTSS1 expression rate in the metastases $(12 \%)$ was lower than that in the primary tumors $(26.5 \%)(\mathrm{P}=0.005)$.

\section{Relationship of loss of MTSS1 expression with poor outcome in patients with gastric cancer}

Figure 2 presents several survival curves of the 669 GC patients, with the median cumulative survival duration in patients with resected gastric carcinoma of 40 months. Kaplan-Meier survival analyses revealed that the GC patients with loss MTSS1 expression had a significantly poor prognosis compared to those with positive MTSS1 expression (18 months versus 76 months; $\mathrm{P}<0.001$, Figure 2A). Furthermore, subgroup analysis of MTSS1 according to TNM was performed (Figure 3A-D). The outcomes of patients with MTSS1-negative expression were worse in each stage than that with MTSS1-positive expression (> 82 months for MTSS1-positive tumors vs 72 months for MTSS1-negative tumors in stage I, P $<0.001$; $>78$ months vs 32 months in stage II, $\mathrm{P}<0.001 ;>70$ months vs 18 months in stage III, $\mathrm{P}<0.001 ;>57$ months vs 11 months in stage IV, $\mathrm{P}<0.001)$. Aside from MTSS1 expression, survival analysis of other clinicopathological factors also revealed that lymph node metastases and clinical TNM stage were associated with prognosis of the patients with gastric cancer (Figure 2B.C).

Multivariate analysis using the Cox proportional hazards model for all of the significant variables in the univariate analysis showed that age, differentiation, nodal invasion, TNM stage and MTSS1 expression were independent prognostic factors (Table 2).

\section{Discussion}

Although metastasis suppressor 1 (MTSS1) may be a critical regulator of carcinogenesis in different cancers, study of MTSS1 has been mainly restricted to several cancers and available data seem to be controversial, leaving the involvement of MTSS1 in cancer not clearly defined. Given that gastric cancer is one of the most malignant cancers in the world and that tumor recurrence and metastases are the major causes of death in patients with gastric cancer, resulting in a poor prognosis of the disease, we set out to investigate the role of MTSS1 in gastric cancer. In this study, we found that loss of MTSS1 expression was significantly associated with important clinical determinants of prognosis for gastric cancer including poorly differentiated tumors, 

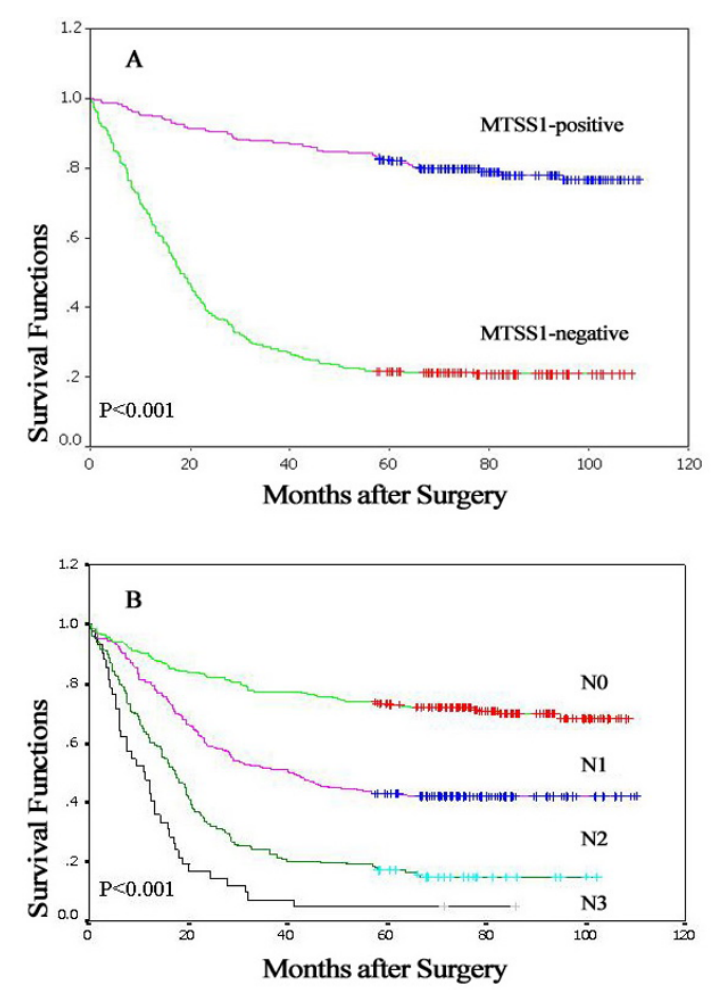

Comparisions and $P$ values

\begin{tabular}{cccc}
\hline N stage & 0 & 1 & 2 \\
\hline 1 & .000 & & \\
2 & .000 & .000 & \\
3 & .000 & .000 & .002 \\
\hline
\end{tabular}

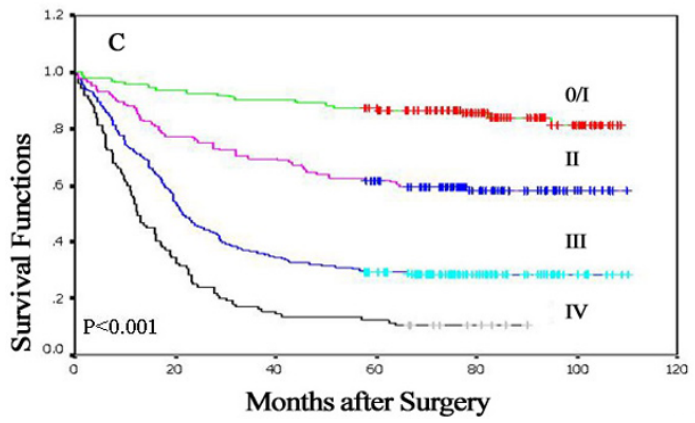

\begin{tabular}{ccccc}
\multicolumn{6}{c}{ Comparisions and P values } \\
\hline TNHI stage & $\mathbf{0}$ & I & II & III \\
\hline I & 0.433 & & & \\
II & 0.203 & .000 & & \\
III & 0.052 & .000 & .000 & \\
IV & 0.010 & .000 & .000 & .000 \\
\hline
\end{tabular}

Figure 2 Kaplan-Meier curves of survival durations in patients with gastric cancer treated with primary gastrectomy. (A) Patients with cancers negative for MTSS1 expression had shorter survival durations than did patients with MTSS1 expression in their gastric cancers $(P<$ 0.001). (B) The survival durations were significantly worse in patients with higher $N$ stages than in those with lower $N$ stages $(P<0.001)$. (C) The survival durations were significantly worse in patients with advanced stages than in those with early stages $(P<0.001)$.

diffuse-type in Lauren's classification, large tumor size, deep invasion level, the presence of nodal metastases and advanced disease stage. And patients without MTSS1 expression had shorter median survival durations than that with MTSS1 expression. Moreover, our immunohistochemistry data clearly revealed that MTSS1 expression significantly decreased from normal tissues to primary tumors to nodal metastases. To the best of our knowledge, this is the first clinical evidence that MTSS1 might play an important role in gastric cancer progression and metastasis.

Accumulating evidences suggested that poorly differentiated tumors have higher growth and recurrence rates than well-differentiated tumors do [17]. Previous study reported that down-regulation of MTSS1 expression might correlate with the transition of tumor cells from distinct epithelium-like morphology to less differentiated carcinomas [18]. Consistently, in our study, MTSS1 protein expression was lost more frequently in poorly differentiated tumors than in well-differentiated tumors, suggesting that MTSS1 is a differentiation marker for gastric cancer. However, another report reveals that an incremental increase in MTSS1 expression was detected from healthy normal liver donors to non tumor tissue specimens and then to their matched hepatocellular carcinoma tumor tissue specimens. Specifically, a 

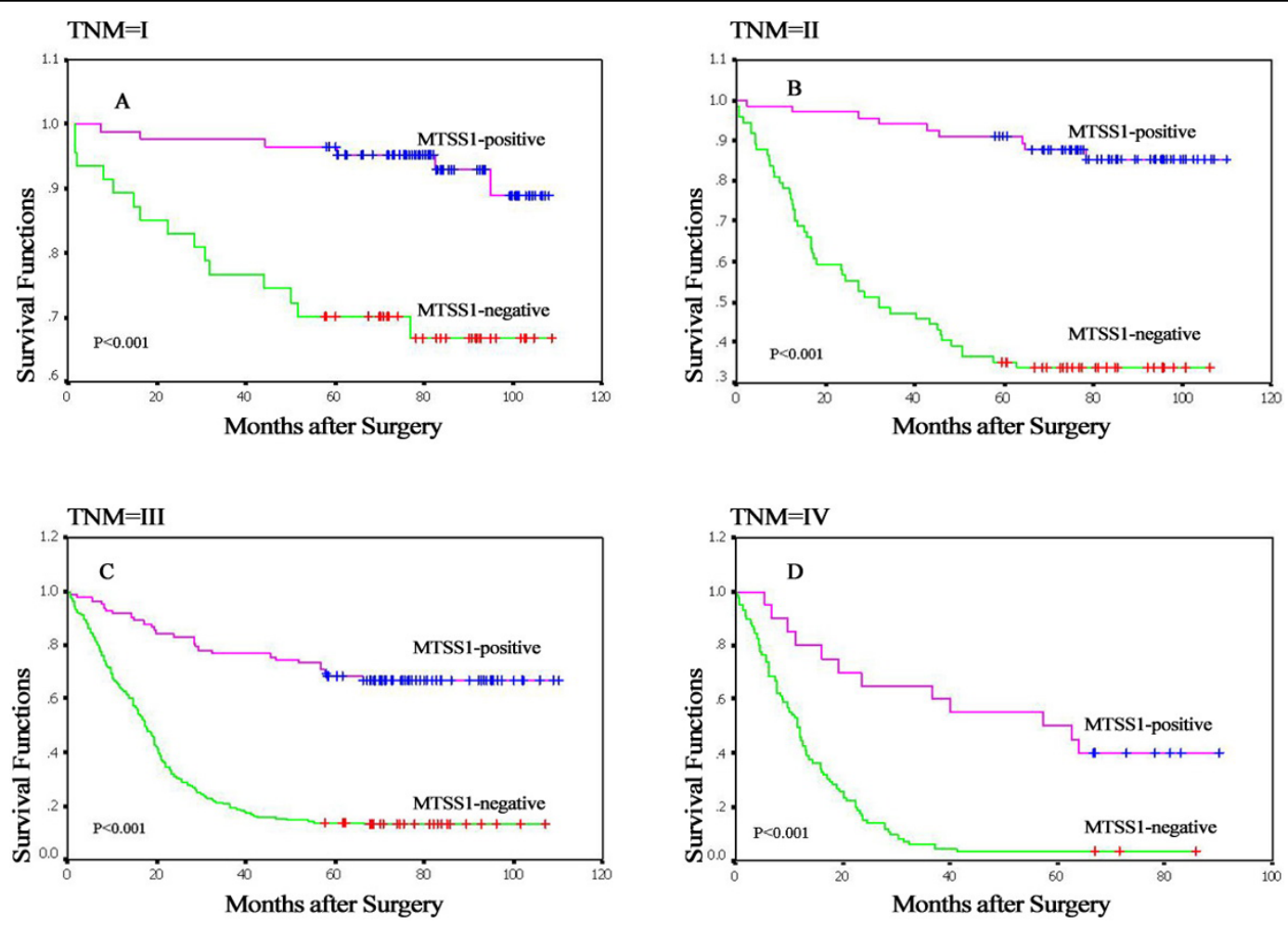

Figure 3 Subgroup analysis of MTSS1 according to TNM. (A-D) Stage-specific survival curves showed patients with loss of MTSS1 expression had poor survival to those with positive MTSS1 expression in each stage $(P<0.001)$.

higher level of MTSS1 expression was observed at the early stages of the disease, suggesting that MTSS1 may play an important role in promoting the early development of hepatocellular carcinoma [16]. Inasmuch as the role of MTSS1 has not been clearly defined to date because of contradicting published data, we speculate that the role of MTSS1 could be cancer or tissue type specific.

Risk factors for gastric cancers have been explored in a number of studies, including status of lymph nodes, depth of tumor invasion, age at diagnosis, TNM stage

Table 2 Cox proportional hazards model analysis of prognostic factors

\begin{tabular}{lccccccc}
\hline & B & SE & Wald & RR & $\mathbf{9 5 \%}$ Cl & $\begin{array}{c}\mathbf{P} \\
\text { value }\end{array}$ \\
\hline Age & 0.416 & 0.109 & 14.652 & 1.516 & 1.225 & 1.876 & $<.001$ \\
Tumor size & 0.107 & 0.119 & 0.800 & 1.112 & 0.881 & 1.405 & 0.371 \\
Differentiation & 0.202 & 0.091 & 4.883 & 1.224 & 1.023 & 1.464 & 0.027 \\
Location & 0.028 & 0.068 & 0.172 & 1.029 & 0.900 & 1.176 & 0.678 \\
Nodal metastasis & 0.288 & 0.093 & 9.588 & 1.334 & 1.112 & 1.601 & 0.002 \\
Gastric wall & 0.267 & 0.144 & 3.409 & 1.305 & 0.984 & 1.732 & 0.065 \\
invasion & & & & & & & \\
TNM & 0.341 & 0.144 & 5.643 & 1.406 & 1.061 & 1.863 & 0.018 \\
MTSS1 & -1.640 & 0.152 & 116.494 & 0.194 & 0.144 & 0.261 & $<.001$ \\
\hline SE, standard & & & & & & &
\end{tabular}

$\mathrm{SE}$, standard error and some molecular markers $[5,7,19-23]$. In the present study, we confirmed that age, histology, nodal metastasis and TNM stage were independent predictors for gastric cancer. Furthermore, MTSS1 expression was found to be significantly correlated with prognosis in univariate survival analysis and it still kept its prognostic value in multivariate survival analysis. Positive associations between MTSS1 expression and other clinicopathologic features, such as tumor size, depth of tumor invasion, status of lymph nodes, TNM stage, differentiation and Lauren's classification were indicated. All these findings suggested that MTSS1 expression alone was a potential molecular marker for predicting outcome in patients who undergo gastrectomy for gastric cancer.

Metastasis is a fatal step in the progression of gastric cancer and has become one of the most challenging problems in tumor therapy [24]. The spread of tumor cells is a complicated and multistage process and requires altered expression of many different genes [25]. Nixdorf et al [13] indicated that down-regulation of MTSS1 occurred in BL17/2 bladder tumor cell lines with invasive abilities. Expression of MTSS1 has also been shown to be reduced in prostate cancer and can contribute to tumor growth and development, as well as metastasis [12]. In this study, we observed significantly lower expression of MTSS1 in the nodal metastases of 
gastric cancers than that in primary tumors. The high frequency of down regulation of MTSS1 expression in primary gastric tumors and lost MTSS1 expression in metastases and their direct association with poor outcome of gastric cancer indicated that MTSS1 could suppress tumor invasion and metastasis, and might be a candidate prognostic factor for lymph node metastasis and tumor progression. This notion is clearly supported by a recent study showing that biological overexpression of MTSS1 significantly suppressed the invasive, migratory, growth and adherence properties of a human breast cancer cell line [26]. However, Bompard et al [15] found that MTSS1 expression is not dependent on the metastatic state of the cells. We also observed MTSS1 expression in six nodal metastases but not in matched primary tumors. This discrepancy makes us presume that there is a possibility that the components which regulate MTSS1 may create specific interactions with different microenvironments between primary regions and target organs. Expression of MTSS1 is regulated by an epigenetic event that is differently represented in different sites. The mechanism for the downregulation of MTSS1 to tumor progression, especially metastasis, is not clear. It was reported that its expression is regulated by DNA methylation, a potential hallmark of a gene likely to be involved in tumor initiation and progression [11]. Additionally, several studies indicated that MTSS1 may be due to actin binding, reorganisation or changes in cell adhesion or tyrosine phosphorylation upon loss of MTSS1 [27]. As reorganization of the actin cytoskeleton has been extensively studied in cancer and is the primary mechanism of cell motility and migration, which are critical steps, involved in tumor carcinogenesis [28]. Further functional investigations are worthwhile to explore the precise mechanism of the carcinogenic effect of MTSS1 with the goal of developing potential therapies targeting MTSS1 as an indicator for gastric cancer.

\section{Conclusion}

To the best of our knowledge, this is the first report demonstrating the clinicopathological significance of MTSS1 expression in gastric cancer. Our results showed that loss of MTSS1 expression was associated with large tumor size, low differentiation, deep invasion level, advanced tumor stage, the presence of nodal metastasis, and poor outcome in patients who underwent gastrectomy. MTSS1 expression may serve as a useful biomarker for the prediction of outcome of gastric cancer.

\section{Acknowledgements}

We thank our team members: Xi Wang, Jun Pan and Zhou Zhou for their selfless assistance with the collection work of clinical data.
This research was supported by grants from Technology Foundation of Jiangsu Health (No: H200844).

\section{Author details}

'Department of Medical Oncology, Changzheng Hospital, Shanghai, China. ${ }^{2}$ Department of Oncology, the Eighty-fifth Hospital, Shanghai, China. ${ }^{3}$ Department of Surgery, the first People's Hospital, Kunshan, Jiangsu, China.

${ }^{4}$ Department of Pathology, Changhai Hospital, Shanghai, China.

\section{Authors' contributions}

$\mathrm{KL}$ participated in the design of the study, carried out the mRNA expression of MTSS1 in GC, the immunohistochemistry of tissue microarrays and drafted the manuscript. GFW participated the immunohistochemistry analysis of MTSS1 in GC patients and assisted the analysis of data. HZD performed the Western Blotting test and assisted the collection of clinical data. YC participated evaluation of immunostaining. GZY and JJW participated in its design and coordination, and supervised the study. KL and GFW contributed equally to this work. All authors read and approved the final manuscript.

\section{Competing interests}

The authors declare that they have no competing interests.

Received: 4 January 2010 Accepted: 15 August 2010

Published: 15 August 2010

\section{References}

1. Parkin DM, Bray F, Ferlay J, Pisani P: Global cancer statistics, 2002. CA Cancer J Clin 2005, 55(2):74-108.

2. Whelan SL: Cancer Incidence in Five Continents. Coding practices. IARC Sci Publ 1992, 120: 31-38.

3. Hill MJ: Mechanisms of gastric carcinogenesis. Eur J Cancer Prev 1994, 3(Suppl 2):25-29.

4. Howson CP, Hiyama T, Wynder EL: The decline in gastric cancer: epidemiology of an unplanned triumph. Epidemiol Rev 1986, 8:1-27.

5. Bozzetti F, Bonfanti G, Morabito A, Bufalino R, Menotti V, Andreola S, Doci R, Gennari L: A multifactorial approach for the prognosis of patients with carcinoma of the stomach after curative resection. Surg Gynecol Obstet 1986, 162(3):229-234.

6. Siewert JR, Bottcher K, Stein HJ, Roder JD: Relevant prognostic factors in gastric cancer: ten-year results of the German Gastric Cancer Study. Ann Surg 1998, 228(4):449-461.

7. Yasui W, Oue N, Aung PP, Matsumura S, Shutoh M, Nakayama H: Molecular-pathological prognostic factors of gastric cancer: a review. Gastric Cancer 2005, 8(2):86-94

8. Lee YG, Macoska JA, Korenchuk S, Pienta KJ: MIM, a potential metastasis suppressor gene in bladder cancer. Neoplasia 2002, 4(4):291-294.

9. Woodings JA, Sharp SJ, Machesky LM: MIM-B, a putative metastasis suppressor protein, binds to actin and to protein tyrosine phosphatase delta. Biochem J 2003, 371(Pt 2):463-471.

10. Pollard TD, Borisy GG: Cellular motility driven by assembly and disassembly of actin filaments. Cell 2003, 112(4):453-465.

11. Utikal J, Gratchev A, Muller-Molinet I, Oerther S, Kzhyshkowska J: The expression of metastasis suppressor MIM/MTSS1 is regulated by DNA methylation. Int I Cancer 2006, 119:2287-2293.

12. Loberg RD, Neeley CK, Adam-Day LL, Fridman Y, St John LN, Nixdorf S, Jackson P, Kalikin LM, Pienta KJ: Differential expression analysis of MIM (MTSS1) splice variants and a functional role of MIM in prostate cancer cell biology. Int J Oncol 2005, 26(6):1699-1705.

13. Nixdorf S, Grimm MO, Loberg R, Marreiros A, Russell PJ, Pienta KJ, Jackson P: Expression and regulation of MIM (Missing In Metastasis), a novel putative metastasis suppressor gene, and MIM-B, in bladder cancer cell lines. Cancer Lett 2004, 215(2):209-220.

14. Callahan CA, Ofstad T, Horng L: MIM/BEG4, a sonic hedgehog-responsive gene that potentiates Gli-dependent transcription. Genes Dev 2004, 18:2724-2729.

15. Bompard G, Sharp SJ, Freiss G, Machesky LM: Involvement of Rac in actin cytoskeleton rearrangements induced by MIM-B. J Cell Sci 2005, 118(Pt 22):5393-5403 
16. Ma S, Guan XY, Lee TK, Chan KW: Clinicopathological significance of missing in metastasis B expression in hepatocellular carcinoma. Hum Pathol 2007, 38(8):1201-1206

17. Yu G, Wang J, Chen Y, Wang X, Pan J, Li Q, Xie K: Tissue microarray analysis reveals strong clinical evidence for a close association between loss of annexin A1 expression and nodal metastasis in gastric cancer. Clin Exp Metastasis 2008, 25(7):695-702.

18. Wang Y, Liu J, Smith E, Zhou K, Liao J, Yang GY, Tan M, Zhan X: Downregulation of missing in metastasis gene (MIM) is associated with the progression of bladder transitional carcinomas. Cancer Invest 2007, 25(2):79-86.

19. Wu CW, Hsieh MC, Lo SS, Tsay SH, Li AF, Lui WY, P'Eng F K: Prognostic indicators for survival after curative resection for patients with carcinoma of the stomach. Dig Dis Sci 1997, 42(6):1265-1269.

20. Shiraishi N, Adachi Y, Kitano S, Bandoh T, Inomata M, Yasuda K, Tsuchihashi J, Kinukawa N: Gastric cancer with extragastric lymph node metastasis: multivariate prognostic study. Gastric Cancer 2000, 3(4):211-218.

21. Zhan YQ, Sun XW, Li W, Chen YB, Xu L, Guan YX, Li YF, Xu DZ: [Multivariate prognostic analysis in gastric carcinoma patients after radical operation]. Ai Zheng 2005, 24(5):596-599.

22. Orsenigo E, Carlucci M, Braga M, Tomajer V, Di Palo S, Tamburini A, Di Carlo V, Staudacher C: [Prognostic factors of gastric neoplasms: experience with 1,074 cases undergoing surgical treatment at a single center]. Supp/ Tumori 2005, 4(3):S86-87.

23. Saito H, Osaki T, Murakami D, Sakamoto T, Kanaji S, Tatebe S, Tsujitani S, Ikeguchi M: Effect of age on prognosis in patients with gastric cancer. ANZ J Surg 2006, 76(6):458-461.

24. Ishii K, Kinami S, Funaki K, Fujita H, Ninomiya I, Fushida S, Fujimura T, Nishimura G, Kayahara M: Detection of sentinel and non-sentinel lymph node micrometastases by complete serial sectioning and immunohistochemical analysis for gastric cancer. J Exp Clin Cancer Res 2008, 27:7.

25. Guan-Zhen Y, Ying C, Can-Rong N, Guo-Dong W, Jian-Xin Q, Jie-Jun W: Reduced protein expression of metastasis-related genes (nm23, KISS1, KAl1 and p53) in lymph node and liver metastases of gastric cancer. Int J Exp Pathol 2007, 88(3):175-183.

26. Parr C, Jiang WG: Metastasis suppressor 1 (MTSS1) demonstrates prognostic value and anti-metastatic properties in breast cancer. Eur $J$ Cancer 2009, 45(9):1673-1683.

27. Lee SH, Kerff F, Chereau D, Ferron F, Klug A, Dominguez R: Structural basis for the actin-binding function of missing-in-metastasis. Structure 2007, 15(2):145-155

28. Zhong C, Kinch MS, Burridge K: Rho-stimulated contractility contributes to the fibroblastic phenotype of Ras-transformed epithelial cells. Mol Biol Cell 1997, 8(11):2329-2344.

\section{Pre-publication history}

The pre-publication history for this paper can be accessed here: http://www.biomedcentral.com/1471-2407/10/428/prepub

\section{doi:10.1186/1471-2407-10-428}

Cite this article as: Liu et al:: Downregulation of metastasis suppressor 1 (MTSS1) is associated with nodal metastasis and poor outcome in Chinese patients with gastric cancer. BMC Cancer 2010 10:428.

\section{Submit your next manuscript to BioMed Central and take full advantage of:}

- Convenient online submission

- Thorough peer review

- No space constraints or color figure charges

- Immediate publication on acceptance

- Inclusion in PubMed, CAS, Scopus and Google Scholar

- Research which is freely available for redistribution

Submit your manuscript at www.biomedcentral.com/submit 